\title{
El comienzo del Feminismo Institucional en el Ayuntamiento de Madrid
}

\author{
The beginning of Institutional Feminism \\ in the Local Government of Madrid
}

\author{
Leticia SEgura ORDAZ * \\ Instituto de Investigaciones Feministas \\ de la Universidad Complutense de Madrid
}

Recibido: 27/11/2019

Aceptado: $16 / 09 / 2020$

doi: https://doi.org/10.20318/femeris.2021.5931

\begin{abstract}
Resumen. Este artículo trata el tema del Feminismo Institucional a nivel municipal a partir de la conformación de la primera Concejalía de Promoción de la Igualdad y Empleo del Ayuntamiento de Madrid en el año de 1999. El análisis se basa en la literatura sobre el tema y en una revisión histórica del proceso mediante entrevistas cualitativas y revisión documental.

Después de dos décadas de la creación de la primera concejalía de igualdad del Ayuntamiento de Madrid, hay pocos estudios hechos relacionados con este acontecimiento. Con el objetivo de ampliar el conocimiento referente al Feminismo Institucional a nivel municipal, el presente artículo pretende identificar las condiciones que facilitaron la creación de la primera estructura de política de igualdad del Ayuntamiento de Madrid, enfocando la atención en los actores que formaron parte del proceso.

Se concluye que los factores más influyentes que permitieron la configuración del Feminismo Institucional en el Ayuntamiento de Madrid fueron: la presencia de actores críticos como el movimiento feminista, el apoyo de partidos políticos de izquierda dentro del gobierno y la influencia de las políticas internacionales y europeas. La presencia de estos actores impulsó la introducción de objetivos feministas en la política municipal de igualdad que dio como resultado la creación del Consejo de Mujeres de la ciudad y de la figura de agentes de igualdad, dos actores que en alianza promovieron el Feminismo Institucional en el Ayuntamiento de Madrid.

Palabras clave: Feminismo Institucional, políticas públicas de igualdad de género, gobierno municipal, Ayuntamiento de Madrid, movimiento feminista.
\end{abstract}

Abstract. This article addresses the issue of Institutional Feminism at municipal level, from conformation of the first structure of equality policies of the Madrid City Council in 1999. The analysis is based on the literature on the subject and on a historical review of the process through qualitative interviews and documentary review.

After two decades of the creation of the first structure of equality policies of the Madrid City Council, there are few studies made related to this case. In order to broaden the knowledge regarding Institutional Feminism at the municipal level, this article aims to identify the conditions and actors that facilitated the creation of the first equality policy structure of the Madrid City Council, focusing attention on the actors who were part of the process.

\footnotetext{
${ }^{*}$ lesegura@ucm.es
} 
It is concluded that the most influential factors that allowed the configuration of Institutional Feminism in the Madrid City Council were the presence of critical actors such as the feminist movement, the support of left-wing political parties within the government and the influence of international and European policies. The presence of these actors prompted the introduction of feminist objectives in the municipal equality policy, which resulted in the creation of the Council of Women of the city and the figure of equality agents, two actors who in the form of an alliance promoted Institutional Feminism at the Madrid City Council.

Keywords: Institutional Feminism, gender equality policies, local government, Madrid City Council, feminist movement.

\section{Introducción}

A partir de 1975, con la celebración de la I Conferencia Mundial sobre las Mujeres de Naciones Unidas, realizada en México y con los consiguientes acuerdos internacionales, los gobiernos de los países miembros de las Naciones Unidas asumen la responsabilidad de llevar a cabo una revisión y establecimiento de normativas y políticas con perspectiva de género. Como consecuencia, la mayoría de los países occidentales comenzaron a establecer organismos encargados de promover la igualdad de género, convirtiéndose en actores clave para defender en todos los niveles de gobierno las demandas de las mujeres en las políticas públicas.

El establecimiento de estos organismos de políticas de igualdad llamó la atención de las académicas para analizar su impacto y funcionamiento, derivando en el análisis de Feminismo Institucional, el cual hace referencia a la capacidad que tiene un gobierno para contrarrestar la desigualdad de género a través del establecimiento de organismos encargados de promover políticas que defiendan intereses feministas, así como la presencia y participación de actores/as feministas en el desarrollo de dichas políticas. La investigación dirigida por Dorothy McBride Stetson y Amy Mazur en 1995 se identifica como uno de los principales análisis sobre Feminismo Institucional que se enfocó en dos criterios, el primero en la influencia política de los organismos de igualdad para promover objetivos feministas en las políticas; y el segundo, en la relación entre estos organismos de igualdad y el movimiento feminista, en específico, en la capacidad del gobierno para desarrollar oportunidades de acceso directo o indirecto a grupos, intereses y actividades feministas en las políticas, todo ello tomando en cuenta para su estudio el contexto social, político e histórico que influyó en la conformación de estructuras de políticas de igualdad.

Sin embargo, hasta ahora la investigación empírica sobre Feminismo Institucional que trata el caso del Estado español, se ha enfocado más en estudios comparativos con otros países occidentales (Mazur, 2009, McBride Stetson y Mazur, 1995; McBride y Mazur 2010; Outshoorn y Kantola, 2007), que en estudios regionales (Alonso Álvarez 2015; Bustelo, 2016: Valiente 1994; 1995) y locales (Alonso Álvarez 2019; Gelambí Torrell, 2015; Lombardo, 2002; Roldán García, 2004; Valiente, 1999), sobre todo si se trata del caso de Feminismo Institucional en el Ayuntamiento de Madrid, el cual no cuenta con estudios 
registrados ${ }^{1}$ a pesar de su importancia, no sólo por su condición de capitalidad y por ser la ciudad con mayor densidad poblacional, sino por contar con una amplia y creciente trayectoria de movilización de mujeres y feministas que contrasta con el extenso recorrido de gobiernos conservadores que han estado a cargo del Ayuntamiento (de 1989 a 2015).

Tomando en cuenta lo anterior, este artículo pretende ampliar el conocimiento sobre el Feminismo Institucional a nivel local, a través del estudio del proceso de creación del primer organismo de política de igualdad del Ayuntamiento de Madrid, con el objetivo de identificar cuáles son las condiciones que permitieron su establecimiento, poniendo especial atención al papel de los/as actores/as; así como en los elementos que coinciden con la literatura especializada en Feminismo Institucional.

Para conseguir el objetivo planteado, este artículo se divide principalmente en cuatro partes, en la primera, se describe brevemente la metodología utilizada para llevar a cabo la investigación. En una segunda sección se hace una aproximación a los primeros análisis llevados a cabo en estudios comparativos a nivel central y regional que han sido referentes en la literatura de género y política, debido a que presentan el análisis de múltiples factores que pueden contribuir a la conformación del Feminismo Institucional. Al mismo tiempo, esta sección se divide en tres segmentos para identificar las principales características que componen el Feminismo Institucional que son: la existencia de una estructura de política de igualdad; la influencia de un movimiento de mujeres; y otros factores que pueden influir en la configuración del Feminismo Institucional.

En la tercera parte se describe el proceso y los factores que dieron inicio al Feminismo Institucional del Ayuntamiento de Madrid, tomando en cuenta el contexto histórico español que dio pie a la creación de la primera Concejalía municipal.

Por último, en la cuarta sección se describe el papel de los/as actores/as que estuvieron presentes e influyeron en la configuración del Feminismo Institucional municipal, como el movimiento feminista, las concejalas de igualdad y las agentes de igualdad.

Finalmente, se hace un breve resumen a manera de conclusión de los hallazgos más relevantes descritos en este trabajo, los cuales se relacionan con el papel de los/as actores/ as involucrados/as en el proceso de establecimiento del Feminismo Institucional madrileño, tales como la presión del movimiento feminista de la ciudad, de las mujeres de los partidos de izquierda que formaban parte de la oposición del gobierno municipal, así como el efecto contagioso de las políticas de igualdad en otros gobiernos multinivel del país.

\subsection{Nota metodológica}

En primer lugar es importante mencionar que este artículo se basa únicamente en el periodo de 1999 a 2003, tiempo en el que se establece y desarrolla la primera Concejalía

\footnotetext{
${ }^{1}$ Es necesario rescatar que uno de los trabajos que ha servido para este artículo, a pesar de no estar publicado, es la Memoria de Grado de Barreiros Ribao, Purificación, titulado "10 años de Políticas de Igualdad en el Ayuntamiento de Madrid: Metaevaluación de las evaluaciones realizadas y diseño de la evaluación de la nueva Estrategia para la Igualdad”, realizada en el marco del Master en Evaluación de Programas y Políticas Públicas en 2010.
} 
de Igualdad del Ayuntamiento de Madrid, debido a que se considera un punto de partida que sentó las bases para el desarrollo del Feminismo Institucional madrileño, así como a la necesidad de contar con referencias que describan este proceso de inicio de las políticas de igualdad municipal que contrasta con el contexto político conservador.

La metodología seguida en esta investigación es de tipo cualitativa y se basa en tres entrevistas en profundidad de tipo semiestructurado; estas entrevistas ayudaron a conformar una línea de tiempo para identificar los principales acontecimientos que fueron detallados con información documental (como el I Plan de Igualdad de Oportunidades entre Mujeres y Hombres 2000-2004, notas de periódicos y Memorias e Informes publicados por el Consejo de las Mujeres del Municipio de Madrid). Cabe señalar que debido a la escasez de referencias sobre el estudio de caso, para dar inicio a la investigación, en una primera etapa, se realizaron dos entrevistas en profundidad en octubre de 2016 a dos agentes de igualdad del Ayuntamiento de Madrid que forman parte del organismo de igualdad desde el año 2000, ya que cuentan con experiencia como personal experto en las políticas de igualdad madrileñas. En una etapa posterior del proceso de investigación, se llevó a cabo una entrevista a una integrante del Consejo de las Mujeres del Municipio de Madrid (CM) en marzo de 2018, que ha formado parte del movimiento feminista de la ciudad desde hace más de tres décadas. El principal objetivo de las entrevistas fue conocer cómo fue creado el primer organismo de igualdad y qué voces se tomaron en cuenta en el proceso. Las entrevistas en profundidad, conducidas personalmente por la investigadora en la ciudad de Madrid, tuvieron una duración aproximada de una hora y fueron transcritas y analizadas por la propia autora ${ }^{2}$.

\section{Aproximación a la literatura sobre Feminismo Institucional}

Como ya se ha mencionado, el Feminismo Institucional hace referencia al estudio de la capacidad que pueden tener los gobiernos para defender intereses feministas en el proceso de decisiones políticas (Lovenduski, 2005, p. 4; McBride Stetson y Mazur, 1995, p. 10). Este concepto parte de cuatro áreas del conocimiento de las ciencias sociales: el institucionalismo; los movimientos sociales; la democratización y representación; y los marcos políticos (McBride y Mazur 2010, p. 6).

Los términos de "Feminismo Institucional" (Lovenduski 2005; Reverter Bañón, 2011), "feminismo de Estado" (Bustelo, 2004; McBride Stetson y Mazur, 1995; Outshoorn y Kantola, 2007; Valiente, 1999), o "femocracias" (Threlfall 1990) han sido utilizados para referirse al establecimiento de estructuras institucionales que tienen entre sus objetivos formular y poner en práctica políticas de igualdad, pero los términos varían según los criterios relacionados con el hecho de que dichas políticas logren promover objetivos fe-

\footnotetext{
${ }^{2}$ Cabe mencionar que este material de investigación forma parte del trabajo de campo de mi tesis doctoral titulada "El Feminismo Institucional en las políticas públicas de igualdad de género del Ayuntamiento de Madrid 1999-2019”, defendida en enero de 2021, en el Instituto de Investigaciones Feministas de la Universidad Complutense de Madrid.
} 
ministas. Es así que Joni Lovenduski (2005) define al Feminismo Institucional como la defensa de las demandas del movimiento de las mujeres dentro del Estado. Mientras que McBride y Mazur (2010) toman en cuenta más elementos para definir que el Feminismo de Estado es "el grado en que las estructuras de políticas de las mujeres forjan alianzas con los movimientos de mujeres y las ayudan a obtener acceso a ámbitos públicos y a alcanzar sus objetivos políticos" (p. 5). La mayoría de los estudios relacionados con este tema coinciden en enfocar el análisis principalmente en el establecimiento y funcionamiento de los organismos de políticas de las mujeres para identificar cuáles son los principales elementos que influyen en la defensa de los intereses feministas por parte del Estado.

El foco del análisis sobre Feminismo Institucional o feminismo de Estado ha ido cambiando de alguna manera. En un inicio estos conceptos se relacionaban únicamente con las personas (sobre todo mujeres) que trabajaban en la administración pública ya sea con un puesto de poder político o aquellas que defendían políticas de género. De acuerdo con Dorothy McBride y Amy Mazur $(1995,2010)$ lo anterior se relacionó con el término de "femócratas", acuñado en la década de 1990 en Australia (Eisenstein 1996; Sawer 1990; Watson 1990) y en los Países Bajos (Outshoorn, 1994). Cabe destacar que la palabra "femócratas" fue utilizada de manera peyorativa para referirse "a las feministas que se habían dejado cooptar, ocupando puestos en los organismos de igualdad" (Bustelo 2004, p. 20), posteriormente esta connotación fue cambiando y en la actualidad se entiende como "las personas que trabajan en los organismos de igualdad, siendo un término que utilizan las propias 'feministas de Estado' para referirse a ellas mismas” (Bustelo, 2004, p. 21). Por otra parte, el término de "femocracias" (Threlfall, 1990) es utilizado para referirse a la creación de estructuras y organismos que se encargan específicamente de impulsar políticas públicas de igualdad (Bustelo 2004).

Posteriormente, la atención cambió hacia la institucionalización del feminismo en organismos de políticas de igualdad y las mujeres que forman parte de dichos organismos (McBride Stetson y Mazur, 1995; Valiente, 1999). Más adelante, diversas investigaciones sobre política feminista comparada (Mazur, 2009; McBride Stetson y Mazur, 1995; Outshoorn y Kantola, 2007) se enfocaron en la representación de los intereses feministas en las políticas públicas y la relación entre los movimientos de mujeres y los organismos encargados de promover la igualdad de género. En otras palabras, estos estudios prestaron principal atención en la capacidad del Estado para contribuir al cumplimiento de una agenda feminista (Lovenduski, 2005, p. 4). El Feminismo de Estado, por lo tanto, no se refiere únicamente a la idea de incluir mujeres en el gobierno, sino a fomentar la participación de actores/as de movimientos sociales de mujeres y feministas para facilitar la inclusión de sus demandas en las políticas públicas.

Dorothy McBride Stetsony Amy Mazur en 1995 se enfocaron especialmente en identificar las condiciones que ayudan a que el Estado promueva la influencia del movimiento de mujeres en las decisiones gubernamentales a través de sus estructuras estatales de políticas de igualdad. Como resultado, está investigación derivaría en diversas publicaciones que analizan la conceptualización del Feminismo de Estado, tomando en cuenta el contexto de los gobiernos democráticos estudiados. 
Posteriormente, con la intención de examinar lo que había ocurrido a más de una década del estudio realizado por McBride Stetson y Mazur en 1995, Joyce Outshoorn y Johanna Kantola, en 2007, editan el libro “Changing State Feminism”, el cual, se centra en las consecuencias de la reconfiguración del Estado que se derivan de la implementación de la transversalidad de la perspectiva de género y la ampliación de las demandas derivadas de los enfoques hacia la diversidad y la interseccionalidad, además de que agregan al análisis la influencia de procesos externos de gobernanza, globalización y regionalización, en la reforma del Estado de bienestar (Lombardo y Verloo, 2010, p. 17).

Por su parte Amy Mazur, en 2009, añade al análisis una aproximación a la medición de la influencia feminista durante todo el proceso de las políticas públicas, prestando especial atención a la representación descriptiva y sustantiva del feminismo que explica Hanna Pitkin ${ }^{3}$. El análisis de Mazur, por lo tanto, buscaba identificar algunos factores que se encontraran presentes en los casos con mayor éxito de representación en el feminismo de Estado. Entendiendo el éxito como la respuesta dual por parte del gobierno al movimiento feminista y de mujeres al incluir sus demandas en las políticas y promover su participación en el proceso.

Los estudios mencionados sobre el análisis de Feminismo Institucional sirven de referencia empírica y teórica para este artículo, y muestran que dos elementos esenciales para analizar el concepto son: la existencia de una estructura encargada de desarrollar políticas públicas de igualdad de género y la influencia de los objetivos del movimiento de mujeres y feministas en el proceso político.

\subsection{Estructura de políticas de igualdad de género}

Si bien es cierto que uno de los elementos principales para hablar de Feminismo Institucional es la presencia de una estructura de gobierno diseñada para promover políticas públicas dirigidas a mejorar las condiciones sociales, económicas y políticas de las mujeres, es necesario no limitar el concepto y estudio de Feminismo Institucional a la simple presencia de estas estructuras (Bustelo, 2016, p. 31). El valor de las estructuras de igualdad en el Feminismo Institucional es que pueden llegar a introducir y defender objetivos feministas en las políticas, y formar un vínculo entre el Estado y los grupos organizados de mujeres, además de que es una oportunidad de acceso para que algunas mujeres trabajen dentro del gobierno, diseñando e implementando proyectos dedicados a mejorar el status de las mujeres (McBride Stetson y Mazur, 1995, p. 12). Para Luvenduski (2008, p. 174) las Women `s Policy Agencies (Agencias de Políticas de Mujeres) son un foro de representación de las mujeres en la toma de decisiones políticas que permiten insertar metas feministas en las políticas públicas. Estas agencias o estructuras de igualdad forman parte del análisis del Feminismo Institucional, sobre todo, porque ayudan a identificar qué tan efectivo

\footnotetext{
${ }^{3}$ La representación descriptiva se refiere a la presencia de actores feministas en el proceso político, mientras que la representación sustantiva es cuando el debate y los resultados políticos tienen en cuenta las demandas feministas (Mazur, 2009, pág. 332)
} 
es el Estado para tomar en cuenta e incluir a las mujeres y sus objetivos (McBride y Mazur, 2010, p. 5).

Las estructuras u organismos de política de igualdad de género pueden presentarse como oficinas, comisiones, agencias, ministerios, comités, secretarías, asesores o áreas (Kantola y Outshoorn, 2007, p.3; McBride Stetson y Mazur, 1995, pp. 2-3;). En el caso de los ayuntamientos, también pueden ser concejalías, un área o dirección general dentro de una concejalía. Celia Valiente (1999, p.176) señala que a nivel municipal, las concejalías se distinguen del resto de organismos, por tener mayor ventaja para realizar políticas de igualdad, debido a que un concejal o concejala al depender directamente de alcaldía y formar parte del equipo de gobierno puede defender personalmente las estrategias diseñadas por esta estructura de igualdad.

Alba Alonso, agrega (2015), que la capacidad de las estructuras de políticas de igualdad depende de sus características que le permitan abordar "el mayor abanico de temas posibles y tener amplias posibilidades de ser eficaz en la promoción de las políticas de género" (p. 54). Por lo tanto, el nivel jerárquico de las estructuras de igualdad en el organigrama establece su capacidad de influencia, así como su estabilidad a lo largo del tiempo, pues facilita que las estructuras de igualdad y los movimientos de mujeres formen vínculos para defender objetivos feministas, pero también puede permitir que los organismos y, sobre todo, que el personal técnico que lo componen adquiera experiencia en la formulación de políticas feministas ${ }^{4}$.

Es necesario aclarar que el trabajo con los objetivos feministas requiere de un proyecto a largo plazo (Masolo, 2007, p. 9) que no puede depender únicamente del establecimiento de estructuras de políticas de igualdad, o de la inclusión de mujeres y del movimiento feminista en el proceso político, sino que requiere de la combinación de diversos factores que se irán detallando más adelante.

\subsection{Movimiento de mujeres y feministas}

Para el Feminismo Institucional el papel de los movimientos de mujeres también es significativo. Amy Mazur (2009, p.350), en su artículo "Les Mouvements Féministes et l'élaboration des Politiques Dans une Perspective Comparative", explica que para hablar de Feminismo Institucional no sólo es necesario enfocar la atención en la existencia de un organismo público que promueva políticas de igualdad, sino también, analizar la participación de los actores del movimiento feminista y la inclusión de sus demandas en las políticas públicas.

Mazur (2009) reconoce que un movimiento de mujeres puede encontrarse tanto fuera como dentro del Estado y presentarse como una organización formal o informal, a través de manifestaciones o presiones, además, señala que cualquier actor individual

\footnotetext{
${ }^{4}$ Se entiende por política feminista aquella que "promociona la situación de las mujeres y/o socavan los patrones de la jerarquía de género” (Bustelo, 2004, p. 22)
} 
"también puede identificarse como actores en un movimiento de mujeres (parlamentarias, expertas o periodistas), siempre que tengan una demanda del movimiento de mujeres." (p. 333) ${ }^{5}$, Sin embargo, distingue que no todas las voces del movimiento de mujeres representan a todas las mujeres, ni que todas son feministas. Los movimientos de mujeres y los movimientos feministas se diferencian según su perspectiva y actuación. A pesar de que ambos se caracterizan por ser un colectivo de mujeres, las reivindicaciones de los movimientos feministas se distinguen por aportar ideas y realizar demandas fundamentadas en teorías feministas, por ello, cuestionan las desigualdades basadas en sus identidades de género como mujeres y sus demandas están dirigidas a lograr un cambio en las relaciones de género. Mientras que el movimiento de mujeres se centra en reivindicaciones para llevar a cabo cambios sociales que no son específicamente feministas (Bustelo y Lombardo, 2016, 435; Ferree y McClory, 2004, p. 476).

El valor de los movimientos feministas y de mujeres es que han logrado influir en el desarrollo de instrumentos de políticas de igualdad, como leyes y planes, además de que han contribuido a promover cambios institucionales dedicados a dichas políticas de igualdad (McBride Stetson y Mazur, 1995, p. 1; Outshoorn y Kantola, 2007, p. 269). En opinión de Incháustegui (1999, p. 103), tanto las organizaciones de mujeres como el movimiento feminista requieren ser incluidos en el análisis del Feminismo Institucional, ya que movilizan diversos recursos para lograr la integración de las demandas de las mujeres para su transformación en interés público a partir de su participación en la formulación de propuestas políticas.

De acuerdo con las analistas de Feminismo Institucional (Mazur, 2009, p. 349; Outshoorn y Kantola, 2007, p. 280) para lograr que el movimiento de mujeres y feministas influya en las agendas de gobierno es necesario que el tejido asociativo de mujeres sea fuerte ${ }^{6}$. La fortaleza del movimiento contribuye a mantener a los propios organismos de políticas de igualdad, debido a que ayudan a ejercer presión sobre las políticas del Estado para innovar e incluir objetivos feministas y su participación en el proceso, y al mismo tiempo inciden en la democratización del proceso político. Los movimientos de mujeres más fuertes pueden llegar a influir en el éxito del Feminismo Institucional (Amy Mazur 2009, p.337). Sin embargo, Alba Alonso (2015, p. 58) menciona que también existe la tendencia a que los movimientos desaparezcan o pierdan su fuerza, debido a la creciente vinculación relacionada con las instituciones que les hagan perder su autonomía; en segundo lugar, a la ausencia de ciertos tipos de protesta o la fragmentación que alcanzan.

McBride y Mazur (2010, 248), encontraron que cuando el movimiento de mujeres se enfrenta a condiciones desfavorables para su éxito como la falta de fuerza o fragmentación, no solo los organismos de políticas, sino además una combinación de otros factores puede contribuir con el éxito de incluir objetivos feministas a las políticas estatales.

Amy Mazur (2009, p. 348), añade al análisis la presencia de actores críticos en el desarrollo de políticas que pueden ayudar a incluir objetivos feministas, como expertas en

\footnotetext{
${ }^{5}$ Las traducciones que se citan en este artículo fueron realizadas por la autora.

${ }^{6}$ Para cuantificar y definir la fuerza de los movimientos las investigaciones discuten en torno a tres características: fragmentación, activismo e institucionalización (véase Mazur, 2009, p. 337)
} 
temas de género, o aliados/as con poder dentro de las instituciones, que pueden o no ser feministas. Por su parte Alison Woodward (2015, pág. 5) se basó en la metáfora del "triángulo de terciopelo", para analizar el papel de los/as actores/as individuales o grupales que interactúan y forman alianzas durante el proceso de políticas públicas de igualdad de género. El concepto de triángulo de terciopelo nace en el 2003 y explica la relación entre las personas encargadas de hacer las políticas (femócratas), las expertas y académicas especialistas en género, y los movimientos de mujeres y feministas. El triángulo de terciopelo puede considerarse como una categoría de análisis importante que ayuda a entender estos procesos de intercambio de información y a valorar el papel de las mujeres como portadoras de ideas fundamentales para el éxito o el fracaso de las políticas de igualdad, una tarea esencial para la conformación del Feminismo Institucional.

\subsection{Posibles factores facilitadores del Feminismo Institucional}

Los estudios sobre política feminista comparada presentan una serie de posibles condiciones que pueden encontrarse en algunos casos de éxito de Feminismo Institucional. No obstante, es necesario aclarar que de acuerdo con las estudiosas del feminismo de Estado (Massolo, 2007; Mazur 2009; McBride Stetson y Mazur, 1995, 2010; Kantola y Outshoorn, 2007), el éxito no depende específicamente de un solo factor sino de la alineación de algunos de ellos, ya que a menudo ciertos factores identificados como importantes no parecen tener un efecto significativo para la institucionalización del feminismo. Los elementos que pueden encontrarse, de acuerdo con los estudios hechos son:

1. La presencia de un gobierno de izquierda (Mazur, 2009, p.337; McBride Stetson y Mazur, 1995, p. 288).

2. Contar con una organización institucional con la capacidad de influir en la formulación de políticas feministas (Mazur, 2009, p. 346; McBride Stetson y Mazur, 1995, p. 288).

3. La concepción del Estado como un posible agente promotor de cambio desde el cual se pueden defender intereses feministas (McBride Stetson y Mazur, 1995, p. 289).

4. La fortaleza y organización del movimiento de mujeres y feministas, con ausencia de divisiones en torno a un tema. Lo cual, significa que a mayor fuerza del movimiento, mayor será la presión que pueden llegar a ejercer en el gobierno (Mazur, 2009, p. 347; McBride Stetson y Mazur, 1995, p. 290).

5. Alianzas entre el movimiento y la estructura de política de igualdad, como un vínculo estratégico para defender y legitimar las políticas feministas (Mazur, 2009, p. 349350).

6. Presencia de actores críticos, ya sea dentro o fuera del Estado, que defiendan los objetivos feministas como aliados del feminismo o como parte del movimiento. Sobre todo las expertas en género y femócratas. (Mazur, 2009, p. 348) 
Finalmente, es necesario que cada contexto en el que se analizan los casos de Feminismo Institucional, prestar atención a la influencia de estos elementos en los cambios a nivel institucional y social. Pero al mismo tiempo, tener en cuenta la influencia de la regulación internacional, principalmente de la Organización de las Naciones Unidas (ONU) y de la Unión Europea (UE), en el establecimiento de estas agencias de igualdad de género. Para Johanna Kantola y Joyce Outshoorn (2007, p. 10-11), la UE, "es una fuente importante de financiamiento que ha mantenido y establecido agencias de política de la mujer", por lo tanto, para estas autoras, la UE representa un tipo de Feminismo Institucional al que llaman $f e$ minismo supraestatal, y al Feminismo Institucional que se encuentra en niveles de gobierno regional y municipal, como feminismo subestatal. Ambos tipos de Feminismo Institucional se caracterizan principalmente por provocar un efecto contagioso y de aprendizaje multinivel para la adopción de políticas de igualdad. La influencia del feminismo upranacional debe considerarse en el análisis sobre la institucionalización del feminismo, sobre todo porque ha representado para las femócratas una herramienta de coacción política para dar cumplimiento a acuerdos internacionales y europeos (Mazur, 2009, p. 348).

\section{Feminismo Institucional en el Ayuntamiento de Madrid}

Una vez identificados los elementos principales que caracterizan el Feminismo Institucional, en este apartado, se examinan los factores que influyeron y que coinciden con la literatura, con el proceso de establecimiento de la primera Concejalía de Promoción de la Igualdad y Empleo y el papel de las y los principales actores/as que influyeron para su establecimiento.

El principal hallazgo de esta investigación es que la creación de la Concejalía de Promoción de Igualdad y Empleo puede calificarse como uno de los acontecimientos históricos más importantes no sólo del movimiento asociativo de mujeres y feministas de la ciudad, sino del Feminismo Institucional del municipio de Madrid, pues uno de los factores más importantes que facilitaron su establecimiento fue la alianza entre actores críticos dentro del Ayuntamiento y el movimiento de mujeres y feministas. En los siguientes apartados se muestran los resultados del análisis de las entrevistas y la revisión documental, pasando por un breve contexto histórico relacionado con las políticas de igualdad en España a nivel central y autonómico que dan sentido al propio proceso que se investiga.

\subsection{Contexto histórico, sobre el Feminismo Institucional Español}

En 1983, se crea en España el Instituto de la Mujer (IM) "seis años después de las elecciones democráticas y un año después de que el Partido Socialista Obrero Español (PSOE) entrara al poder por primera vez" (Valiente, 1995, p. 221). Celia Valiente (1995, p. 224), destaca que el factor que impulsó la creación del IM fue el apoyo de feministas del propio partido, quienes argumentaron la necesidad de contar con un Instituto de la Mujer y con políticas 
de igualdad para formar parte de la Unión Europea, que a pesar de que sucede hasta 1986, la entrada se fue preparando años antes. A partir de entonces, se empiezan a elaborar medidas e instrumentos de políticas de igualdad como leyes y planes. En comparación con otros países europeos, España experimentó uno de los patrones de crecimiento más rápidos, ya que de una dictadura, pasó a una democracia moderna impulsora de políticas públicas y marcos institucionales orientados a la igualdad de género (Bustelo y Ortbals, 2007, pág. 202).

A finales de 1980, aproximadamente cinco años después de la creación del IM, las Comunidades Autónomas (CCAA), siguiendo el modelo del gobierno central, empezaron a crear políticas de igualdad de género que contenían iniciativas para ser implementadas por distintos organismos gubernamentales. Para María Bustelo (2016, pág. 34), la característica que más destaca de este proceso es la diversidad de las actuaciones de las CCAA a la hora de desarrollar políticas, discursos y estructuras para promover la igualdad.

En 1989, seis años después de la creación del IM, la Comunidad Autónoma de Madrid (CAM) establece la Dirección General de la Mujer. Posteriormente, a principios de los años noventa, algunos municipios de la CAM establecieron las primeras concejalías de la mujer imitando a otros organismos de igualdad de distintos niveles gubernamentales (Valiente, 1999, p. 177). María Bustelo y Candice Ortbals (2007, p. 213) señalan que la formación de estos espacios se debe también al impacto de las políticas de la Unión Europea, las cuales proporcionaron un marco legal que dio legitimidad a las demandas de las mujeres, además de que contribuyó al desarrollo y creación de organismos de igualdad.

De acuerdo con Emanuela Lombardo (2002, p. 82-83) tanto a nivel nacional como autonómico existe el compromiso de tener en cuenta las líneas estratégicas establecidas por la ONU y la UE al momento de legislar y diseñar políticas públicas de igualdad, mientras que a nivel municipal, al ser más cercanos a la población, es posible prestar servicios que sean más adecuados a sus necesidades. Esta autora, identifica que los gobiernos locales tienen las bases legales para desarrollar políticas de igualdad en la Ley del Régimen Local y en los artículos 14 y 9.2 de la Constitución Española que establecen, por un lado la igualdad jurídica y por otro la obligación de los poderes públicos para facilitar la igualdad efectiva entre las personas.

\subsection{La creación de la Concejalía de Promoción de Igualdad y Empleo}

El comienzo de lo que podemos llamar Feminismo Institucional en el Ayuntamiento de Madrid, se da en 1999 cuando se crea la primera Concejalía de Promoción de la Igualdad y Empleo, que permanece durante cuatro años hasta 2003 después de un cambio de gobierno municipal. Para entender los factores que influyeron en el establecimiento de la primera estructura encargada del desarrollo de políticas de igualdad madrileñas es necesario analizar primero los antecedentes de estas políticas, tomando en cuenta dos actores fundamentales del objeto de estudio: el gobierno municipal y el movimiento feminista.

Después de la transición a la democracia los primeros mandatos del Ayuntamiento de Madrid, de 1979 a 1989, estuvieron a cargo del PSOE. El análisis de las entrevistas pone 
de relieve que esta época muestra un primer acercamiento por parte de algunas mujeres que trabajaban dentro del Ayuntamiento para desarrollar políticas dirigidas a promover la participación de las mujeres. Sin embargo en 1989, como consecuencia de una moción de censura que dejó como alcalde a Agustín Rodríguez Sahagún del partido Centro Democrático y Social (CDS), el enfoque de las políticas públicas municipales tuvo un carácter más conservador que detuvo el avance de las políticas dirigidas a las mujeres, y que se acentuó en los años noventa durante el gobierno de José María Álvarez del Manzano del Partido Popular, impidiendo por lo tanto la institucionalización de las políticas de igualdad (Agente de igualdad 1).

El gobierno de José Álvarez del Manzano se mantuvo durante tres mandatos municipales (1991-2003) de los cuales, tanto el primero como parte del segundo, a pesar de contar con una Comisión de la Mujer ${ }^{7}$, representaron un periodo de especial retroceso para las políticas de igualdad con la reducción de servicios de atención a mujeres víctimas de violencia, de cuidado de la infancia y recortes de becas de comedor. Otra característica de este periodo es que el alcalde no era partidario de entablar un diálogo con las asociaciones de mujeres de los barrios de la ciudad, pese a la frecuente insistencia por parte de estas asociaciones para solicitar al Ayuntamiento la puesta en marcha de políticas de igualdad (San José Serrano, 1995, p. 63).

A pesar de que tradicionalmente España se caracteriza por una débil relación entre el movimiento feminista y el Estado, y que en los ayuntamientos de la CAM el movimiento no fue relevante para la creación de concejalías (Bustelo, 2016; Valiente, 1999), los antecedentes del movimiento feminista que influiría en el Feminismo Institucional madrileño se relacionan con la Plataforma de Mujeres de la Ciudad de Madrid, un grupo conformado por 69 entidades organizadas de mujeres de los distintos barrios y las áreas de mujeres de los sindicatos (Aguirre, 2000). Este grupo, puede señalarse como uno de los actores que promovió el establecimiento de la primera estructura de políticas de igualdad en el Ayuntamiento debido a su constante presión al gobierno municipal. En 1991, se presentan por primera vez en el Pleno del Ayuntamiento para demandar la creación de una Concejalía que representara a las mujeres, y un año después, en 1992, deciden agregar una serie de demandas que serían secundadas por las mujeres de los partidos opositores que formaban parte del Pleno Municipal, es decir, los partidos de Izquierda Unida (IU) y PSOE. Durante siete años, esta lista fue actualizada y demandada al gobierno municipal en conmemoración del Día Internacional de la Mujer. Algunas de las principales demandas incluidas en dicha lista fueron, además de la creación de la Concejalía encargada de políticas de igualdad, el desarrollo de un Plan de Igualdad municipal, la creación de un Consejo de participación para las mujeres, el establecimiento de "Casas de la mujer" y la contratación de agentes de igualdad en los 21 Distritos de la ciudad (Aguirre, 2000; San José Serrano, 1995; Agente de igualdad 1).

${ }^{7}$ De acuerdo con Begoña San José (1995), esta Comisión únicamente se dedicó a recabar informes sobre la carrera administrativa de mujeres y hombres en el Ayuntamiento, entre otras cosas que no fueron relevantes para la conformación de políticas de igualdad municipal 
De acuerdo con el análisis de las entrevistas realizadas, es posible identificar tres factores que explican el interés de la Plataforma de Mujeres de la Ciudad de Madrid para solicitar la creación de la Concejalía, los cuales son:

1. La falta de políticas de igualdad. Si bien algunas femócratas intentaron promover la participación de las mujeres, la falta de apoyo del gobierno del PP para impulsar actuaciones formales dirigidas hacia las mujeres es un factor que impulsó el trabajo de presión del movimiento feminista hacia el Ayuntamiento.

2. El auge de organismos de igualdad en la década de los años noventa. Como se muestra en la sección anterior sobre el contexto histórico, después de la creación del IM en 1983, el establecimiento de agencias dedicadas a las políticas de igualdad se popularizó en España, siendo la década de los 90 cuando los Ayuntamientos instauran las primeras Concejalías de la Mujer, lo cual despertó el interés de la Plataforma de Mujeres y de los partidos PSOE e IU para solicitar la Concejalía.

3. La confianza en el gobierno municipal como un agente de cambio y defensor de los intereses feministas. La Plataforma de Mujeres reconocía la necesidad de empezar a formar parte de las decisiones que se tomaban en la ciudad, tomando en cuenta que el Ayuntamiento es la institución más cercana a las mujeres (Integrante del CM).

Es importante resaltar que el apoyo de los grupos políticos municipales de izquierda al movimiento asociativo de mujeres representa uno de los factores que impulsó el Feminismo Institucional madrileño. La presencia de lo que Mazur (2009) llama "actores críticos" o "aliados con poder dentro de la institución" que apoyan la causa feminista, resultan ser un elemento significativo para el proceso de creación de la concejalía y políticas de igualdad en el Ayuntamiento de Madrid. Un factor que también estuvo presente a nivel Estatal en el establecimiento del Instituto de las Mujeres que fue impulsado por iniciativa de las mujeres del propio partido (PSOE) al frente del gobierno (Bustelo, 2016).

Otro factor que explica la creación de la Concejalía de Promoción de la Igualdad y Empleo fue la influencia del Feminismo Institucional supraestatal y subestatal. Las agentes de igualdad entrevistadas coincidieron en señalar que el efecto contagioso de la puesta en marcha de políticas de igualdad en los gobiernos multinivel del país, así como la influencia de las políticas internacionales y europeas influyeron en el cambio de idea de Álvarez del Manzano para apoyar el desarrollo de políticas específicas dirigidas hacia las mujeres. En 1997, el gobierno central de José María Aznar, también presionado por cumplir y hacer cumplir los acuerdos internacionales de las mujeres, instruyó al alcalde la necesidad de atender los acuerdos firmados con Naciones Unidas, tales como la IV Conferencia Mundial sobre la Mujer realizada en Beijing en 1995, la Convención sobre la Eliminación de toda forma de Discriminación contra la Mujer (CEDAW) aprobada en 1979, y sobre todo las recomendaciones y directivas de la UE en materia de igualdad como la recomendación del Consejo del 2 de diciembre de 1996 relativa a la participación equilibrada de mujeres y de hombres en la toma de decisiones (96/694/CE). 
Derivado de lo anterior, el Ayuntamiento de Madrid llevó a cabo un diagnóstico para identificar las políticas que se dirigían a las mujeres. El diagnóstico, arrojó como resultado que distintas áreas municipales contaban con programas diseñados para la atención de mujeres (en aspectos de estricta marginación social) y con presupuesto. Sin embargo, también puso de manifiesto la falta de una tradición en políticas de sensibilización en igualdad de género en la atención a la ciudadanía y, sobre todo, se dieron cuenta que no existía una política que englobara la atención a las mujeres. Con la elaboración de este diagnóstico el Ayuntamiento concluyó, en primer lugar, que era necesario facilitar el acercamiento con el movimiento asociativo de mujeres y feministas para conocer sus propuestas y acordar una línea de actuación, a través de un departamento o un lugar para desarrollar políticas de igualdad y, en segundo lugar, la necesidad de contar con un documento que agrupara todas las acciones dirigidas a las mujeres para proporcionar mayor visibilidad y constancia de la existencia de dichas acciones (Agente de igualdad 1)

Sin embargo, a principios de los años noventa, mientras que el movimiento asociativo de la ciudad de Madrid solicitaba al Ayuntamiento espacios de participación para reivindicar sus derechos, en el resto de los municipios de la CAM algunas estructuras de igualdad ya habían comenzado a crearse. Por otro lado, de acuerdo con Celia Valiente (1999, p. 178) las Concejalías de algunos municipios no se establecieron de cero, sino que se apoyaron de los procesos que ya se hacían desde los organismos de servicios sociales, y en este caso, el Ayuntamiento de Madrid no fue diferente de esta regla, ya que también se apoyó del Área de Servicios Sociales para elaborar el diagnóstico y conocer el desarrollo de las políticas que se dirigían hacia las mujeres.

Otro elemento que explica la conformación del Feminismo Institucional del Ayuntamiento de Madrid es la fuerza institucional con la que se establece la primera estructura de políticas de igualdad, ya que la existencia de una concejalía específica de igualdad refleja la atención que se le da a las políticas de igualdad (Roldán García, 2004, p. 126). María Bustelo (2004, p. 29) considera que un organismo de igualdad es fuerte si se toman en cuenta dos factores: el tipo de la estructura de igualdad que se crea y el departamento o área gubernamental donde se encuentra. En este caso, el establecimiento de esta estructura como una Concejalía que dependía directamente del alcalde y no como una Dirección representa una oportunidad política para defender los intereses del movimiento de mujeres y feministas en las políticas públicas. Sin embargo, el hecho de que esta Área de Gobierno comparta su marco de atención política al denominarse como "Promoción de la Igualdad y Empleo", debilita su valor como maquinaria institucional de políticas de igualdad ya que no permite garantizar que los objetivos feministas sean el eje dominante del trabajo de este organismo ${ }^{8}$.

\footnotetext{
${ }^{8}$ Vale la pena profundizar en futuros análisis, ¿qué significado se da a la igualdad en la política diseñada por esta estructura? y ¿cuáles son los marcos dominantes? con el objetivo de determinar cómo afecta la atención de las políticas de igualdad y por lo tanto al Feminismo Institucional el hecho de que una concejalía se presente con un enfoque multicausal.
} 


\section{Actores presentes y su papel en el Feminismo Institucional}

Como hemos visto, el proceso de creación de la Concejalía de Igualdad y Empleo resulta atractivo en especial porque involucró la participación y alianza entre los/as actores/as feministas tanto dentro como fuera del Ayuntamiento. Sin embargo, es revelador para este análisis, enfocar el estudio en el papel que desarrollaron cuatro tipos de actores que formaron parte del inicio del Feminismo Institucional madrileño, los cuales fueron: los/as actores/as críticos/as que apoyan la promoción de la igualdad desde el Ayuntamiento, las agentes de igualdad y el Consejo de Mujeres.

\subsection{Actores críticos, los partidos de izquierda y las concejalas.}

El apoyo por parte de los partidos políticos PSOE e IU, pueden señalarse como actores críticos que en alianza con la Plataforma de Mujeres, presionaron al Ayuntamiento de Madrid para que institucionalizara y desarrollara políticas de igualdad. Si bien la literatura respecto al Feminismo Institucional encuentra que un partido de izquierda al frente del gobierno tiene mejor disposición para promover políticas sociales y sobre todo políticas feministas, también es cierto, que tanto Celia Valiente en 1999 (p. 177), como Amy Mazur en el 2009 (p.350) encontraron algunas excepciones en sus estudios, con actuaciones interesantes relacionadas con la creación de organismos de igualdad en gobiernos conservadores. Tal es el caso del Ayuntamiento de Madrid, donde al momento de la creación de la concejalía de igualdad, el partido al frente del gobierno estaba representado por el Partido Popular, el cual no tuvo un papel trascendental en el proceso pero permitió su establecimiento. Además de que los grupos políticos municipales de izquierda, a pesar de no estar al frente del gobierno, sino en oposición, resultaron ser actores importantes, como aliados del movimiento feminista para demandar la creación de la Concejalía.

Posteriormente al establecimiento de la Concejalía de Igualdad, otros actores críticos que ayudaron a consolidar la institucionalización de políticas feministas en el Ayuntamiento de Madrid son las concejalas que estuvieron al frente de este organismo. Durante el primer año Dolores Flores estuvo al frente de la Concejalía, siendo sustituida por María Antonia Suárez, quien fue la encargada de este organismo los siguientes tres años. El análisis de las entrevistas pone de manifiesto que ambas concejalas facilitaron la participación del movimiento de mujeres y feministas en el desarrollo de las políticas del Área de igualdad.

El papel de Dolores Flores fue importante para impulsar los intereses del movimiento de mujeres y feministas en las políticas municipales, debido a que contaba con experiencia al haber sido subdirectora del IM y sobre todo, por haber impulsado un enfoque participativo en la elaboración del primer Plan de Igualdad de Oportunidades entre Mujeres y Hombres (I PIOMH 2000-2004), que facilitó la inclusión de las demandas del movimiento de la ciudad.

El análisis de los datos pone de relieve el papel que desempeñó María Antonia Suárez como concejala, que a pesar de no ser militante feminista, no solo apoyó las demandas 
del movimiento asociativo y buscó el consenso para dar continuidad al desarrollo de políticas de igualdad, sino que además impulsó el desarrollo de actuaciones solicitadas por el movimiento. El apoyo de esta concejala destaca, sobre todo, si tomamos en cuenta que en los mandatos anteriores no era posible instrumentar políticas específicas hacia las mujeres debido a la gran resistencia a las demandas del movimiento.

Ambas concejalas, como femócratas, forman parte de una de las puntas del triángulo de terciopelo como defensoras de la introducción de objetivos feministas en las políticas de igualdad. Como responsables políticas, lograron mantener un vínculo con el movimiento de mujeres que sirvió para intercambiar información y trabajar en conjunto en defensa de los intereses del movimiento de mujeres y feministas de la ciudad. El papel que desempeñaron tanto Flores como Suarez se explica como decisivo para impulsar el Feminismo Institucional en Madrid. Ambas se mantuvieron en comunicación con el movimiento de mujeres, para facilitar que el primer Plan de Igualdad fuera consensuado e incluyera la mayoría de las demandas de la Plataforma de Mujeres y de los partidos de izquierda, tales como: la creación de un Consejo de Mujeres de la ciudad, Espacios de Igualdad en cada Distrito; así como la contratación de agentes de igualdad. Por lo tanto, se pone de manifiesto que la presencia de actoras clave y las alianzas con el movimiento de mujeres y feministas son factores que impulsan el Feminismo Institucional, pero al mismo tiempo muestra que el nivel local es un escenario que facilita el acercamiento con el movimiento y la conformación de dichas alianzas.

\subsection{Agentes de igualdad}

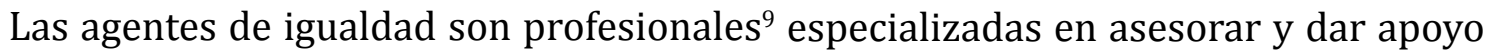
técnico en materia de igualdad de oportunidades entre mujeres y hombres en cada Distrito del municipio ${ }^{10}$. Como personal especializado tienen un papel fundamental para la implementación de la perspectiva de género en las políticas municipales y forman parte del triángulo de terciopelo al representar al conocimiento experto. Al mismo tiempo, al encontrarse dentro de la institución y distribuidas en todo el territorio, les permite conocer directamente la problemática de las mujeres de la ciudad, lo que las posiciona como facilitadoras de la comunicación entre el gobierno local y el movimiento feminista, un factor presente en la literatura para hablar de Feminismo Institucional.

${ }^{9}$ Para ser Agente de Igualdad, se requiere de una titulación o diplomado y contar con experiencia laboral en la administración pública o en la iniciativa social, preferentemente en asociaciones feministas (Agentes de Igualdad 1).

${ }^{10}$ Las funciones de las Agentes han ido evolucionando a lo largo del tiempo para adecuarse a los cambios del propio enfoque de la política de igualdad municipal. Es decir, de dedicarse a llevar a cabo políticas específicas para las mujeres en los Distritos, con la ampliación tanto del enfoque de la política de igualdad como de la Red de Espacios de Igualdad en los Distritos, las agentes se enfocaron en la implementación del mainstreaming de género en el Ayuntamiento, mientras que los Espacios se dedicaron a las políticas específicas dirigidas a las mujeres. 
Debido a la necesidad de contar con apoyo especializado en temas de género en cada uno de los barrios de la ciudad, como ya se tenía en otros municipios, parte de las demandas del movimiento fue solicitar la contratación de agentes de igualdad. En el año 2000 nace en el Ayuntamiento la figura de agente de igualdad, a pesar de que no se crea con una categoría profesional se contratan con carácter temporal a las primeras nueve agentes para trabajar en cinco Distritos. En noviembre del mismo año se acuerda en el Pleno la creación de 21 plazas de agentes de igualdad. Un año después, con el objetivo de contar con el personal técnico especializado que trabajara directamente con la ciudadanía, la plantilla crece a 23 agentes $^{11}$ y se instaura la Coordinación de Agentes de Igualdad. Sin embargo, dichas contrataciones no contaron con un reconocimiento formal de la categoría, ya que se realizó a través de un organismo autónomo y sin estabilidad laboral. Este suceso refleja el compromiso real del gobierno municipal con las políticas de igualdad, ya que la creación de un organismo de igualdad de poco sirve si el personal especializado y encargado de promover la igualdad de género carece de formalidad institucional y por lo tanto, de legitimidad y fuerza para defender y promover los intereses feministas en la política general.

La figura de las Agentes de Igualdad se califica como pieza fundamental para el desarrollo de los Planes de Igualdad del gobierno local, es decir de la política de igualdad a nivel municipal, ya que, al ser el personal del Ayuntamiento, más cercano a la ciudadanía, conocen de manera directa las demandas e intereses de las mujeres; y sirven de acompañamiento para que otras entidades incluyan el enfoque feminista en su trabajo, al mismo tiempo que personifican el interés por institucionalizar y poner en marcha políticas con perspectiva de género (Agente de igualdad 1). Por lo tanto, las agentes, representan un puente de comunicación estratégico entre el movimiento y el Ayuntamiento, y una red de actores especializados con la capacidad de continuar desarrollando conocimiento empírico sobre Feminismo Institucional a nivel municipal.

La contratación de estas especialistas facilitó que las políticas del Ayuntamiento se dirigieran a la promoción de la igualdad, mediante el empoderamiento de las mujeres y la concienciación de la ciudadanía respecto a las desigualdades de género entre mujeres y hombres, y más tarde para implementar la transversalidad de la perspectiva de género. Gracias al análisis de las entrevistas se pone en valor el papel de las agentes de igualdad, debido a que permiten que la perspectiva de género se institucionalice, en este caso, ayudaron a que un gobierno conservador e históricamente contrario al discurso feminista comenzara a tomar en cuenta los objetivos del movimiento de mujeres y feministas en la política municipal.

\subsection{Consejo de Mujeres (CM)}

La creación del CM formó parte de las demandas de la Plataforma de Mujeres desde un inicio. Esta demanda tenía como objetivo contar con un órgano de participación para

${ }^{11}$ En 2004 las agentes de igualdad se distribuyeron en diferentes dependencias de atención a las mujeres, servicios sociales, cultura e igualdad y empleo. Y en la actualidad existen 34 agentes de igualdad. 
las asociaciones de mujeres de la ciudad que sirviera para dar seguimiento a las acciones y medidas que desarrollara el gobierno local en materia de igualdad. A pesar de que dicha demanda se incluyó en el I PIOMH (2000-2004), la creación del CM fue aprobada hasta el 22 de mayo del 2002 en el Pleno del Ayuntamiento por unanimidad de votos.

En esta primera etapa del Feminismo Institucional, la creación del Consejo representó el acceso a la participación del movimiento asociativo de mujeres y feministas en todas las etapas de las políticas del gobierno local, especialmente en el proceso de diseño y evaluación del I PIOMH (2000-2004), lo cual, se traduce en una representación descriptiva del movimiento asociativo tanto en el desarrollo como en los objetivos de estas políticas.

Para María Jesús Rodríguez $(2012,124)$, los Consejos de Mujeres representan un espacio de participación que permiten mejorar la calidad democrática a nivel local, y facilitan la incorporación de un sector de las mujeres, para hacer visibles sus demandas, sobre todo las referentes con políticas de igualdad. Los Consejos de Mujeres según Ramírez, al ser promotores de la participación del movimiento de mujeres permiten la configuración de vínculos estratégicos relacionados con temas de género que repercuten en el desarrollo de políticas.

Los estudios sobre Feminismo Institucional a nivel central (Bustelo 2016, p.4; Bustelo y Ortbals, p. 222;) encontraron que en España, la relación entre los organismos de igualdad y las organizaciones feministas ha sido débil, con algunas colaboraciones en los planes de igualdad a través de los Consejos de Mujeres, pero también encontraron que dicha relación dependía del contexto, aumentando la comunicación a nivel regional y local, debido a la cercanía con la sociedad. Por lo tanto, se reconoce que la política de igualdad del Ayuntamiento de Madrid inició dando un gran paso al favorecer la participación y el asociacionismo de las mujeres en el municipio, lo cual ayudaría a facilitar la alianza entre el movimiento de mujeres y feministas y la Concejalía de Igualdad.

\section{Elaboración del I Plan de Igualdad entre Mujeres y Hombres}

Los organismos de igualdad como responsables de defender objetivos feministas se encargan de desarrollar planes de igualdad como principales instrumentos de política. Un plan de igualdad establece los objetivos y medidas para promover la igualdad de género en un tiempo determinado (Bustelo, 2004, p. 34), que en el caso de los ayuntamientos suele ser de cuatro años. Los planes municipales de igualdad presentan una visión estratégica y un compromiso con la ciudadanía para corregir la desigualdad de género e involucra a otras Áreas y actores municipales para su implementación; mientras que para su elaboración requiere del conocimiento y experiencia del personal experto en igualdad de género y de otras Áreas; además, para el Feminismo Institucional es preferible que incluya la participación del movimiento de mujeres y feministas. Sin embargo, el alcance de los planes de igualdad depende del tipo y posición en el organigrama de la estructura de igualdad que respalde la legitimidad y la capacidad de su personal para incidir con las medidas de igualdad. 
A principios del año 2000, mientras que los planes de igualdad nacional y autonómicos estaban ya en su tercera edición, el Ayuntamiento de Madrid anuncia por primera vez la elaboración de un plan de igualdad municipal (Aguirre, 2000). Este suceso representa un elemento significativo del Feminismo Institucional, ya que el diseño del plan dependió de un proceso participativo que incluyó al movimiento organizado de mujeres y feministas del municipio, impulsando la conformación de una coalición entre actores del movimiento asociativo y el personal responsable y técnico de la Concejalía de Igualdad, entre ellas la concejala. Por lo tanto, contar con la participación del movimiento asociativo en este proceso político representa un factor importante del Feminismo Institucional, pues facilitó que las políticas de igualdad pudieran incluir las demandas de la Plataforma de Mujeres.

El proceso participativo que permitió dar voz a las asociaciones de mujeres para la elaboración del I PIOMH (2000-2004), se explica, debido a que Dolores Flores, como concejala, valoró la necesidad de contar con la participación de los grupos organizados de mujeres para trabajar en conjunto y conocer sus demandas. Posteriormente, María Antonia Suarez retomó la idea de Flores y llevó a cabo una consulta al movimiento de mujeres y feministas, logrando reunir 125 propuestas de 46 organizaciones sociales, con las cuales se elaboró un borrador del plan que posteriormente sería revisado por la Plataforma de Mujeres de la ciudad (Agente de igualdad 2). La consulta dio como resultado que las demandas del movimiento se incluyeran en la elaboración de I PIOMH (2000-2004), que fue aprobado por unanimidad en Acuerdo Plenario del Ayuntamiento el 30 de noviembre de 2000, lo cual significa que contó con el respaldo de todos los partidos políticos. Este Plan se valora principalmente por ser el resultado de un arduo trabajo en conjunto entre el personal de la Concejalía y el tejido asociativo de mujeres, y por representar la configuración del inicio del Feminismo Institucional en el Ayuntamiento de Madrid.

\section{Conclusiones}

En este artículo se llevó a cabo una revisión sobre la primera etapa del feminismo institucional del Ayuntamiento de Madrid con el objetivo de ampliar el conocimiento sobre el Feminismo Institucional a nivel local, a través del análisis del proceso de creación de la primera Concejalía de Promoción de la Igualdad y Empleo se identificaron las condiciones que permitieron su establecimiento, y se prestó especial atención al papel de los actores involucrados, así como los elementos que coinciden con la literatura especializada en Feminismo Institucional.

La revisión de la literatura permitió identificar que el Feminismo Institucional hace referencia a la capacidad que tiene un organismo de gobierno para promover políticas que defiendan objetivos del movimiento feminista. Como se explicó, estos análisis proponen algunos factores que pueden ayudar al establecimiento de dichos organismos de igualdad y a la defensa de las demandas del movimiento, los cuales fueron contrastados en esta investigación y ponen de relieve la importancia de la presencia de actoras del movimiento de mujeres y feministas tanto dentro como fuera del gobierno. Este estudio también 
muestra que el ámbito local es un escenario que facilita la participación y alianza de las actoras del triángulo de terciopelo para impulsar intereses feministas, que en combinación con otros factores actuaron a pesar del contexto político conservador en el que fue creada la Concejalía de Igualdad. Los factores más influyentes en el proceso de establecimiento del feminismo institucional madrileño fueron:

- En primer lugar, la influencia de las políticas internacionales y europeas, así como el efecto contagioso de la puesta en marcha de políticas de igualdad en los gobiernos multinivel, una tendencia del feminismo institucional español.

- En segundo lugar, se encuentra la presencia y presión constante de la Plataforma de Mujeres de la ciudad, un factor que se distingue del resto de las Concejalías establecidas en la CAM, las cuales no contaron con el apoyo del movimiento feminista, y también contrasta con la tendencia en España de la débil relación entre el movimiento y el Estado.

- En tercer lugar, la presencia de actores críticos como las concejalas y su voluntad política para promover la participación del movimiento asociativo de mujeres y feministas en la elaboración del primer plan, así como el apoyo y presión de las mujeres de los partidos de izquierda a las demandas del movimiento.

- En cuarto lugar, se encuentra la alianza entre el movimiento y la agencia de igualdad. Los vínculos que se formaron en este proceso son resultado de la consolidación del Feminismo Institucional a nivel municipal, ya que la creación del Consejo y de la figura de agentes de igualdad, son respuesta a las demandas y objetivos feministas que formaron parte de la política de igualdad del Ayuntamiento.

- Finalmente, a pesar de que el Ayuntamiento de Madrid crea esta estructura con retraso en comparación con el resto de los ayuntamientos de la Comunidad Autónoma de Madrid, su comienzo se caracteriza por ser a gran escala, ya que en dos años se crea la concejalía, se dota de personal especializado, se elabora el Plan de Igualdad y se crea el CM.

\section{Bibliografía}

Alonso Álvarez, Alba (2015). El Mainstreaming de género en España. Hacia un compromiso transversal con la igualdad. Valencia: Tirant lo Blanch.

Alonso Álvarez, Alba (2019). ¿Por las mujeres con las mujeres? Políticas de género participativas en el Ayuntamiento de Barcelona, Política y Sociedad, 56(2), pp. 473-493.

Bustelo, María (2004). La evaluación de las Políticas de género en España. Madrid: Catarata.

- (2016). "Three Decades of State Feminism and Gender Equality Policies in Multi-Governed Spain". Sex Roles 74:107-120. DOI:10.1080/1554477X.2016.1198211. Published first online June $15^{\text {th }}, 2014$. 
Bustelo, María y Lombardo, Emanuela (2016). Género y Política. En M. Barreda, L. Riz Rodríguez, y coordinadores, Análisis de la Política. enfoques y herramientas de la ciencia política (págs. 427-448). Barcelona: HUYGENS.

Bustelo, María y Ortbals, Candice D. (2007). The Evolution of Spanish State Feminism: a Fragmented Landscape. En J. Outshoorn, y J. Kantola, Changing State Feminism (págs. 201-223). London: Palgrave Macmillan.

Eisenstein, Hester (1996). Inside Agitators: Australian Femocrats and the State. Philadelphia: Temple University Press.

Ferree, Myra Marx y McClory, Carol (2004). Feminism and the Women`s Movement: A Global Perspective. En D. Snow, S. Soule, y H. Kriesi, The Blackwell companion to Social Movements (págs. 576-607). UK: Blackwell Publishing.

Gelambí TorRell, Mònica (2015). La gestión transversal de género en momentos de crisis. Análisis de los municipios catalanes. Gestión y Análisis de Políticas Públicas. 1(14), 44-54.

HERnández Rodríguez, GERARdo (1992). El aborto en España, análisis de un proceso "social-político". Madrid: Universidad Pontificia Comillas.

INCHÁUSTEGUI, TERESA (1999). La institucionalización del enfoque de género en las políticas públicas. Apuntes en torno a sus alcances y restricciones. La Ventana (10), 85-123.

Kantola, Johanna y Outshoorn, Joyce (2007). Changing State Feminism. En J. Outshoorn, y J. Kantola, Changing State Feminism (págs. 1-19). London: Palgrave MacMillan

Lombardo, Emanuela (2002). Políticas de igualdad de género en los Ayuntamientos de Barcelona: origen, características, y retos para el futuro. Gestión y Análisis de Políticas Públicas (25): 79-93.

Lovenduski, Joni (2008). State Feminism and Women's Movements. West European Politics, 31, 169-194.

- (2005). State Feminism and Political Representation. Cambridge: Cambridge University Press.

McBride Stetson, Dorothy y Mazur, Amy G. (1995). Introduction. In D. McBride Stetson, A. G. MAzur y Editors, Comparative State Feminism (pp. 1-21). London: SAGE.

McBride, Dorothy y Mazur, Amy (2010). The Politics of State Feminism. Innovation in Comparative Research. Philadelphia, Pennsylvania, United States of America: Temple University Press

MAzur, Amy. G. (2009). Les mouvements féministes et l'élaboration des politiques dans une perspective comparative. Vers une approche genrée de la démocratie. Revue française de science politique, 59(2), 325-351.

Outshoorn, Joyce y Kantola, Johanna (2007). Changing State Feminism. London: Palgrave Mac Millan.

REvERTER BAÑón, Sonia (2011). Los retos del Feminismo Institucional. Revista Internacional de Filosofía (4), 223-229.

RodríGuez GARcía, MARÍA Jesús (2012). Los Consejos Locales de la Mujer en municipios españoles. diagnóstico sobre sesgos de inclusividad y agenda. Revista Española de Ciencia Política (29), 107-128. 
RoldÁn García, Elena (2004). Género, Políticas Locales e Intervención social. Análisis de los servicios de bienestar social municipal para la población femenina en España. Madrid: Complutense.

SAn José Serrano, Begoña (1995). Los ayuntamientos y la igualdad de oportunidades. Cuadernos de Relaciones Laborales, 57-66.

SaWer, Marian (1990). Sisters in Suits, Women and Public Policy in Australia. Sydney: Allen and Unwin.

Threlfall, MóniCA (1990). ¿Patriarca, palanca, paraguas? planteamientos feministas en torno al Estado asistencial. En J. Astelarra, Participación política de las mujeres. 215233.

VAliEnte, Celia (1994). El feminismo de estado en España: El Instituto de la Mujer 19831994. Revista Internacional de Sociología, 163-204.

- (1995) The Power of Persuasion. The Instituto de la Mujer in Spain. En D. McBride Stetson, y A. Mazur, Comparative State Feminism (págs. 221-236). London: Sage Publications.

- (1999). Feminismo de Estado en los ayuntamientos de la Comunidad Autónoma de Madrid. Descentralización de servicios sociales y sanitarios (13-14), 173-189.

Watson, Sophie (1990). Playing the State: Australian Feminist Interventions. London: Verso.

WoodWARD, Alison (Juanuary de 2015). Travel Triángles and transformations: Implication for new agendas in gender equality. Tijdschrift for Gender Studies, 18(1), 5-10.

\section{Documentos consultados en páginas WEB}

AguirRe, BEgoÑa (17 de enero, 2000). El Ayuntamiento prepara el plan de igualdad de la mujer exigido por la oposición desde 1992. El País. Recuperado de: https://elpais. com/diario/2000/01/17/madrid/948111860_850215.html

Consejo de las Mujeres DEl Municipio DE MADRID (2012). “10 Años participando y creando igualdad", Recuperado en: http://www.consejomujeresmadrid.org/wp-content/ uploads/2013/08/intro10anios.pdf

Jiménez, Antonio (31 de mayo, 2000). El alcalde crea una concejalía para un hombre afín a Mercedes de la Merced. El País. Recuperado de https://elpais.com/diario/2000/05/31/madrid/959772255_850215.html

I Plan de Igualdad de Oportunidades entre Mujeres y Hombres 2000-2004, Recuperado de https://www.madrid.es/UnidadesDescentralizadas/IgualdadDeOportunidades/ Publicaciones/Hist\%C3\%B3rico\%20Planes\%20de\%20Igualdad/I\%20PLAN\%20 DE\%20IGUALDAD.pdf 